\title{
The Modulation of a Dust Ion-Acoustic Wave in a Collisional Dusty Plasma
}

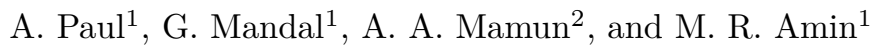 \\ ${ }^{1}$ Department of Mathematics and Physical Sciences, East West University, Aftabnagar, Dhaka 1212, Bangladesh \\ ${ }^{2}$ Department of Physics, Jahangirnagar University, Savar, Dhaka 1342, Bangladesh \\ Email: apual@ewubd.edu
}

\begin{abstract}
The modulation instability of a dust ion-acoustic (DIA) wave in a highly collisional dusty plasma is studied theoretically. In this investigation, effects of ionization, ion loss, and electron superthermality on the DIA wave are included. By employing the standard reductive perturbation technique, a modified-nonlinear Schödinger equation (mNLSE) is developed for the evolution of the slowly varying amplitude of the DIA wave. A detailed analysis of the linear and nonlinear dispersions of the DIA wave is presented. Relevant to some astrophysical objects with typical parameters, it is found that the DIA wave is modulationally unstable below a certain critical wavenumber. Effects of the electron superthermality as well as the ionization and ion loss in the wave dynamics are also studied. It is found that the electron superthermality has a significant effect on the nonlinearity as well as on the damping of the DIA wave. It is also found that the critical wavenumber for the modulation instability is highly dependent on the parameter $\kappa$, known as the spectral index of the electron superthermality. Numerical results on the linear and nonlinear dispersions of the DIA wave are described. A parameter study on the Rouge wave solution of the mNLSE is also presented.
\end{abstract}

Keywords: Dust ion-acoustic (DIA) wave, collisional dusty plasma, superthermality of electron, nonlinear Schödinger equation, modulation instability.

\section{Introduction}

In space plasmas, the presence of dust particles leads to different wave properties than in normal electronion plasmas. For this reason, in the recent few years, dusty plasma has become a very interesting research area both in the theoretical as well as experimental point of views $[1,2,3,4,5,6,7,8,9,10,11,12,13]$. Dusty plasma is considered to be the admixture of electrons and ions with an additional component of dust particles. Most of the current dusty plasma research is on the new aspects of collective interactions and wave propagation. Charging of the dust particles are either positive or negative, depending on the background plasma and the dust-particle charging processes. The charged dust particles in dusty plasmas can significantly change the normal properties of plasma modes both in unmagnetized and magnetized plasmas $[14,15,16,17,18,19,20,21,22]$. Theoretically, two types of acoustic modes are supported by unmagnetized dusty plasmas, namely, dust acoustic (DA) wave involving mobile dust particles [17], and dust ion-acoustic (DIA) wave involving both mobile ions and mobile dust particles [18]; and the existence of these wave modes was later experimentally verified by Barkan et.al. $[7,8]$. Though the linear properties of the DA and DIA waves in dusty plasmas are now completely understood both theoretically and experimentally, but the nonlinear propagation of these waves are not fully understood. The propagation of DA and DIA waves has received a great deal of interest to understand the features of localized perturbations in space and laboratory dusty plasmas $[23,24,25,26]$.

In a recent study, Mayout et. al. [27], have shown the effects of ionization and ion loss on DIA wave in a collisional dusty plasma with suprathermal electrons. They have considered a one-dimensional (1D) DIA wave propagation in the dusty plasma and have derived a set of fully nonlinear equations. By considering the combined effects of ionization, ion loss, and electron suprathermality in the collisional dusty plasma, finally, the authors of reference [27] have derived a modified Korteweg-de Vries (KdV) equation to describe the dynamics of the DIA wave. These authors have studied both compressive and rarefactive DIA waves and have not considered the amplitude modulation of these waves. As far as we know, the effects of electron suprathermality, ionization and ion loss as well as collisional effects on the amplitude modulation of DIA wave have not been considered previously. 
Motivated by Mayout et. al. [27], in the present paper, we consider a model for the 1D propagation of a DIA wave pulse in a collisional dusty plasma with the inclusion of the electron suprathermality as well as ionization and ion loss. We include a viscous damping term in the momentum balance equations due to collisions of the dust and the ion components of the dusty plasma. Along with the linear properties of the wave, we also study the amplitude modulation of the slow evolution of the dissipative DIA wave pulse by deriving a modified nonlinear Schrlodinger equation (mNLSE). We employ the standard reductive perturbation technique [28] to derive the mNLSE for the propagation of the DIA wave in the collisional dusty plasma. We also discuss the possible modulation instability of the electrostatic wave amplitude and perform some parameter studies.

The paper is organized as follows. Section 2 describes the hydro-dynamical model of the collisional dusty plasma and presents the set of highly nonlinear equations for the evolution of the large amplitude DIA wave pulse. In Sec. 3, a mNLSE is derived by employing the reductive perturbation technique. The solution of the derived mNLSE and a nonlinear dispersion relation and hence an expression for the growth rate of the modulation instability of the dissipative DIA wave is derived in Sec. 4. Results are discussed in Sec. 5 along with graphical representation. Finally, Sec. 6 concludes the paper.

\section{The Mathematical Model}

We consider a 1D collisional unmagnetized dusty plasma with negatively charged mobile dust grains, consisting of superthermal electrons, inertial positively charged ions and immobile/stationary background neutral particles. We consider in this dusty plasma situation, ionization and ion losses are present. Different types of collisions: such as ion-neutral, ion-dust, and dust-neutral are expected in this collisional dusty plasma. Here, $-e,-Z_{d} e$, and $e$ are charges of electron, dust grain, and ion respectively. The quantities $m_{j}$ and $n_{j}$ are the mass and the number density of plasma species $j$ respectively. The subscripts $j=e, i, d$ denote respectively the electrons, ions, and dust grains. The nonlinear dynamics of the DIA wave is described by the following set of equations in the normalized form [27]:

$$
\begin{gathered}
\frac{\partial N_{d}}{\partial T}+\frac{\partial}{\partial X}\left(N_{d} V_{d}\right)=0 \\
\frac{\partial V_{d}}{\partial T}+V_{d} \frac{\partial V_{d}}{\partial X}=\beta_{d} \frac{\partial \Phi}{\partial X}-\nu_{d n} V_{d}, \\
\frac{\partial N_{i}}{\partial T}+\frac{\partial}{\partial X}\left(N_{i} V_{i}\right)=Q_{i}-Q_{L}, \\
\frac{\partial}{\partial T}\left(N_{i} V_{i}\right)+\frac{\partial}{\partial X}\left(N_{i} V_{i}^{2}\right)=-N_{i} \frac{\partial \Phi}{\partial X}-\sigma_{i} \frac{\partial N_{i}}{\partial X}-\nu_{i d} N_{i}\left(V_{i}-V_{d}\right)-\nu_{i n} N_{i} V_{i}, \\
\frac{\partial^{2} \Phi}{\partial X^{2}}=\frac{1}{\delta}\left[\left(1-\frac{\Phi}{\kappa-\frac{3}{2}}\right)^{1 / 2-\kappa}-\delta N_{i}+(\delta-1) N_{d}\right],
\end{gathered}
$$

where $\sigma_{i}=T_{i} / T_{e}, \delta=n_{i 0} / n_{e 0}$, and $\beta_{d}=Z_{d} m_{i} / m_{d}$. Here $T_{j}$ and $n_{j 0}$ are respectively the temperature and the equilibrium number density of plasma species $j$. The first term, in square bracket, of the right-hand side of Eq. (5) indicates normalized number density of suprathermal electron [27], where $\kappa$ is the spectral index (usually $\kappa>3 / 2[27]$ ). The electrostatic potential $\Phi$ is normalized by $T_{e} / e$, the dust fluid velocity $V_{j}$ is normalized by the ion-acoustic speed $C_{i}=\left(T_{e} / m_{i}\right)^{1 / 2}$, and the particle number density $N_{j}$ is normalized by its equilibrium value $n_{j 0}$. The time variable is normalized by the ion-plasma period $\omega_{p i}^{-1}=\left(4 \pi e^{2} n_{i 0} / m_{i}\right)^{-1 / 2}$ and the space variable is normalized by the ion-Debye length $\lambda_{D i}=\left(T_{e} / 4 \pi e^{2} n_{i 0}\right)^{1 / 2}$. The last term $\nu_{d n} V_{d}$ in the right-hand side of Eq. (2) represents the frictional 
drag acting on the dust particles which arises during the collisions between dust and neutral particle at frequency $\nu_{d n}$. Similarly, the last two terms in the right-hand side of Eq. (4) represent the frictional drags on the ions during ion-dust and ion-neutral collisions at frequencies $\nu_{i d}$ and $\nu_{i n}$ respectively. The term $Q_{L}\left(=\nu_{L} N_{i}\right)$ in Eq. (3) accounts for ion loss at frequency $\nu_{L}$. Ion-electron recombination and absorption of plasma particles by the dust grains are mainly responsible for this ion loss.

The term $Q_{i}\left(\approx \nu_{L}\left[1+\left(\Delta \sigma / \sigma_{0}\right) \Phi\right]\right)$ in Eq.(3) accounts for new ion creation through ionization of the neutral gas by the fast suprathermal electrons, where $\sigma_{0}$ and $\Delta \sigma=(d \sigma / d \Phi)_{0}$ are respectively the ionization cross-section and its derivative with respect to the electrostatic potential $\Phi$ at $\Phi=0$ [27]. It is noted that the following terms $\nu_{i d}, \nu_{i n}, \nu_{d n}$, and $\nu_{L}$ are normalized by the ion-plasma frequency $\omega_{p i}$ and $Q_{i}$ is normalized by $\omega_{p i} n_{i 0}$.

\section{Derivation of the Modified Nonlinear Schrödinger Equation}

We apply the reductive perturbation technique [28] to derive the mNLSE, by introducing the following stretched coordinates: $\xi=\epsilon\left(X-v_{0} T\right)$ and $\tau=\epsilon^{2} T$, where $v_{0}$ is a constant and will be determined later from the compatibility condition of the reductive perturbation analysis and $\epsilon$ is a smallness parameter $(0<\epsilon<1)$ that measures the weakness of the dispersion. Now, we expand the dependent variables $N_{j}$, $V_{j}$, and $\Phi(j=d, i)$ in the power series of $\epsilon$ :

$$
\begin{gathered}
N_{j}=1+\sum_{n=1}^{\infty} \epsilon^{n} \sum_{l=-\infty}^{\infty} N_{j l}^{(n)}(\xi, \tau) e^{i l(k X-\omega T)}, \\
V_{j}=\sum_{n=1}^{\infty} \epsilon^{n} \sum_{l=-\infty}^{\infty} V_{j l}^{(n)}(\xi, \tau) e^{i l(k X-\omega T)}, \\
\Phi=\sum_{n=1}^{\infty} \epsilon^{n} \sum_{l=-\infty}^{\infty} \Phi_{l}^{(n)}(\xi, \tau) e^{i l(k X-\omega T)}
\end{gathered}
$$

where $\omega$ and $k$ are respectively the angular frequency and the wavenumber of the carrier DIA wave. Experimental situations show that the normalized collision frequencies are very small, therefore, we can use [29]: $\bar{\nu}_{d n}=\nu_{d n} \epsilon^{2}, \bar{\nu}_{L}=\nu_{L} \epsilon^{2}, \bar{\nu}_{i d}=\nu_{i d} \epsilon^{2}$, and $\bar{\nu}_{i n}=\nu_{i n} \epsilon^{2}$, where $\nu_{d n}, \nu_{L}, \nu_{i d}$, and $\nu_{i n}$ are finite quantities but less than unity. The quantities $N_{j l}^{(n)}(\xi, \tau), V_{j l}^{(n)}(\xi, \tau)$, and $\Phi_{l}^{(n)}(\xi, \tau)$ are the $l-$ th harmonic of the $n$-th order slowly varying field variables and these variables satisfy the reality condition $A_{-l}^{(n)} \equiv A_{l}^{(n) *}$, where the asterisk denotes complex conjugation. We mentioned that the stretched variables and the expanded variables in Eqs. (6)-(8) are employed and accordingly we have two coordinate systems: $(X, T)$ coordinate system, in which there is a rapid variation of the field quantities of the carrier wave with wavenumber $k$ and angular frequency $\omega$; and the $(\xi, \tau)$ coordinate system, in which there is a slowly modulated wave packet. If we now substitute the expansions of $N_{j}, V_{j}$ and $\Phi$ of field variables from Eqs. (6)-(8), into the set of Eqs. (1)-(5), we can easily get the $l$-th harmonic of the $n$-th order reduced equations. Listing of those reduced equations are not shown here as they are very lengthy and readers also will not be much curious.

If we consider the first harmonic of the first order quantities $(n=1$ and $l=1)$, in the reduced equations, the following linear dispersion relation of the DIA wave in the collisional dusty plasma is easily obtained:

$$
k^{2}\left[\frac{\delta}{\omega^{2}-k^{2} \sigma_{i}}+\frac{\beta_{d}(\delta-1)}{\omega^{2}}-\delta\right]=\alpha,
$$

where $\alpha=(\kappa-1 / 2) /(\kappa-3 / 2)$, and the parameters $\delta, \sigma_{i}$, and $\beta_{d}$ are defined earlier. From the first harmonic of the second order quantities $(n=2$ and $l=1)$, and using Eq. (9), we obtain the following 
compatibility condition:

$$
\begin{aligned}
\frac{(\delta-1)}{\omega}\left(v_{0} a_{1}-a_{2}\right) & +\frac{k(\delta-1)}{\omega^{2}}\left(v_{0} a_{2}+\beta_{d}\right) \\
& -\frac{k \delta}{\omega^{2}-k^{2} \sigma_{i}}\left(v_{0} a_{4}-\sigma_{i} a_{3}-1\right)-\frac{\omega \delta}{\omega^{2}-k^{2} \sigma_{i}}\left(v_{0} a_{3}-a_{4}\right)=2 k \delta,
\end{aligned}
$$

where $a_{1}=-k^{2} \beta_{d} / \omega^{2}, a_{2}=-k \beta_{d} / \omega, a_{3}=k^{2} /\left(\omega^{2}-k^{2} \sigma_{i}\right)$, and $a_{4}=k \omega /\left(\omega^{2}-k^{2} \sigma_{i}\right)$. The above compatibility condition, Eq. (10), gives the expression for the group velocity $v_{0}$ as:

$$
\begin{aligned}
v_{0} & =\left[2 k \delta-\frac{k\left(a_{3} \sigma_{i}+1\right) \delta}{\omega^{2}-k^{2} \sigma_{i}}-\frac{\omega a_{4} \delta}{\omega^{2}-k^{2} \sigma_{i}}-\frac{k \beta_{d}(\delta-1)}{\omega^{2}}+\frac{\omega a_{2}(\delta-1)}{\omega^{2}}\right] \\
& \times\left[-\frac{k a_{4} \delta}{\omega^{2}-k^{2} \sigma_{i}}-\frac{\omega a_{3} \delta}{\omega^{2}-k^{2} \sigma_{i}}+\frac{k a_{2}(\delta-1)}{\omega^{2}}+\frac{\omega a_{1}(\delta-1)}{\omega^{2}}\right]^{-1} .
\end{aligned}
$$

It would be wise to mention here that, we are particularly interested in the lower-frequency limit of the dust ion-acoustic wave mode for longer wavelength perturbation $(\omega, k)$.

Here, we are not going through a detailed derivation of the mNLSE, but a brief procedure is stated. From the $l=2$ part of the second order $(n=2)$ reduced equations, the second order harmonic mode $\Phi_{2}^{(2)}$ of the carrier wave is obtained in terms of $\Phi_{1}^{(1)} \Phi_{1}^{(1)}$, where $\Phi_{1}^{(1)}$ is the first order mode, obtained in deriving the linear dispersion relation, Eq. (11) of the DIA wave. The mode $\Phi_{2}^{(2)}$ comes from the nonlinear self-interaction. Similarly, from the $l=0$ part of the third order $(n=3)$ reduced equations, the zeroth harmonic mode $\Phi_{0}^{(2)}$ is obtained in terms of $\left|\Phi_{1}^{(1)}\right|^{2}$. Its expression cannot be obtained from the second order equations. Finally, substituting the expressions for $\Phi_{2}^{(2)}$ and $\Phi_{0}^{(2)}$ into the $l=1$ component of the third order $(n=3)$ part of the reduced equations, we finally obtain the following mNLSE for the slow evolution of the dissipative DIA wave:

$$
i \frac{\partial a}{\partial \tau}+P \frac{\partial^{2} a}{\partial \xi^{2}}+Q|a|^{2} a=-i R a,
$$

where $a \equiv \Phi_{1}^{(1)}$ and

$$
\begin{gathered}
P=\left[\delta+g_{1} b_{4}+g_{2} b_{3}-g_{3} b_{2}-g_{4} b_{1}\right]\left[a_{1} b_{4}+a_{2} b_{3}-a_{3} b_{2}-a_{4} b_{1}\right]^{-1}, \\
Q=\left[b_{1} f_{4}+b_{2} f_{3}-b_{3} f_{2}-b_{4} f_{1}-2 \beta h\right]\left[a_{1} b_{4}+a_{2} b_{3}-a_{3} b_{2}-a_{4} b_{1}\right]^{-1}
\end{gathered}
$$

and

$$
R=\left[a_{2} b_{3} \bar{\nu}_{d n}+b_{1}\left(a_{2}-a_{4}\right) \bar{\nu}_{i d}-a_{4} b_{1} \bar{\nu}_{i n}-b_{2}\left(a_{3}-\Delta \sigma / \sigma_{0}\right) \bar{\nu}_{L}\right]\left[a_{1} b_{4}+a_{2} b_{3}-a_{3} b_{2}-a_{4} b_{1}\right]^{-1} .
$$

The parameters $b_{1}, b_{2}, b_{3}$, and $b_{4}$ are given by $b_{1}=k \delta /\left(\omega^{2}-k^{2} \sigma_{i}\right), b_{2}=\omega \delta /\left(\omega^{2}-k^{2} \sigma_{i}\right), b_{3}=k(\delta-1) / \omega^{2}$, and $b_{4}=\omega(\delta-1) / \omega^{2}$. The other parameters $f_{1}, f_{2}, f_{3}, f_{4}, g_{1}, g_{2}, g_{3}, g_{4}$ and $h$ appearing in Eqs. (13)-(15) are listed in the Appendix A.

The coefficients $P$ and $Q$ appearing in the mNLSE, Eq. (12), are known as dispersion coefficient and nonlinear coefficient respectively. The signs of $P$ and $Q$ determine whether the slowly varying wave amplitude is modulationally stable or not. If the signs of $P$ and $Q$ are such that $P Q<0$ then the wave amplitude is modulationally stable and the corresponding solution of the mNLSE is called a dark soliton [30] when the dissipation $R$ is considered as zero. But, if $P Q>0$, then the wave amplitude may be modulationally unstable and the solution of the mNLSE is called a bright soliton [30] if the dissipation $R$ is considered as zero. It is remarkable that whether dark soliton or bright soliton appears, it is only when the right-hand side of Eq. (12) is zero, i.e., in the situation when there is no dissipation. On the other hand, in the viscous-case $(R \neq 0)$, i.e., if there is some dissipation in the system, the mNLSE does not admit any stationary envelope solitonic solution. However, we are particularly interested about the modulation instability of the nonlinear DIA wave in the longer wavelength limit, and this will be discussed later. 


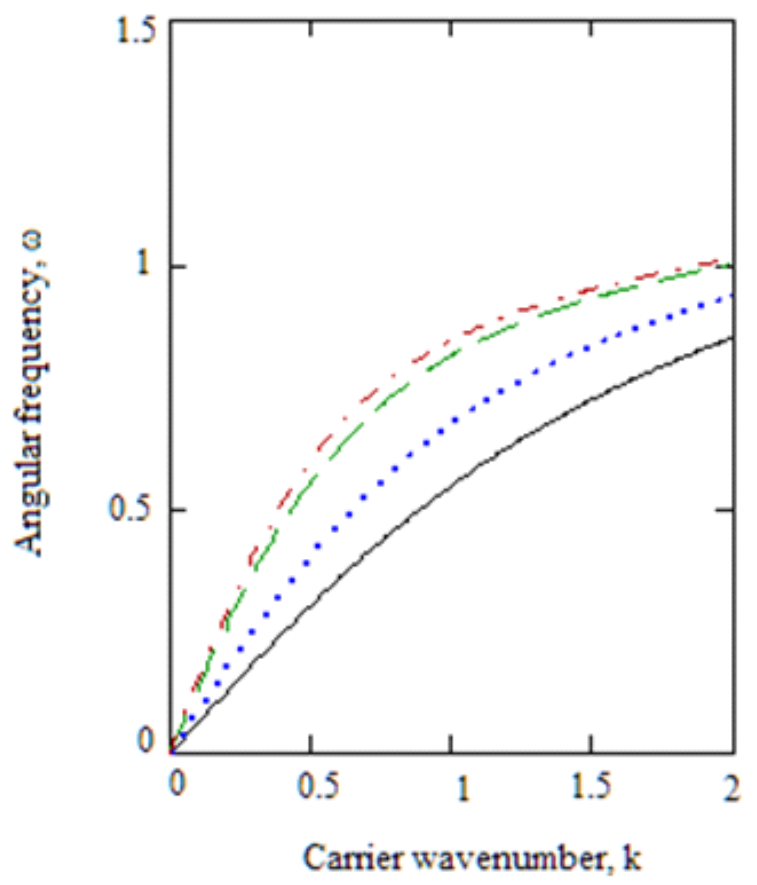

Figure 1. Variation of the angular frequency $\omega$ with respect to the propagation wavenumber $k$ of the dust ion-acoustic wave for different values of the spectral index $\kappa$. The solid curve is for $\kappa=1.7$, the dotted curve is for $\kappa=2$, the dashed curve is for $\kappa=5$, and the dot-dashed curve is for $\kappa=50$. The other parameters of the plasma are the following: $\delta=n_{i 0} / n_{e 0}=2.2, \sigma=0.033, \beta_{d}=3.75 \times 10^{-6}$, and $\bar{\nu}_{i d}=0, \bar{\nu}_{i n}=0, \bar{\nu}_{d n}=0, \bar{\nu}_{L}=0$.

\section{Solution of the mNLSE and Modulation Instability of the DIA Wave}

Here, we present the dissipative nonlinear DIA wave solution of the mNLSE, Eq. (12), within the instability region, where the signs of the dispersion coefficient $P$ and the nonlinear coefficient $Q$ of the mNLSE are same, i.e., $P Q>0$. The required solution can be easily obtained by substituting $a(\xi, \tau)=\Phi_{0}(\xi, \tau) \exp (-R \tau)[31]$ into Eq. (12), we get:

$$
i \frac{\partial \Phi_{0}}{\partial \tau}+P \frac{\partial^{2} \Phi_{0}}{\partial \xi^{2}}+e^{-2 R \tau} Q\left|\Phi_{0}\right|^{2} \Phi_{0}=0 .
$$

Since the damping (dissipative) coefficient $R$ is very small, so by the Taylor expansion, we have $e^{-2 R \tau} \approx$ $1 /(1+2 R \tau)$. Using this approximation, Eq. (16) can be written as

$$
i \frac{\partial \Phi_{0}}{\partial \tau}+P \frac{\partial^{2} \Phi_{0}}{\partial \xi^{2}}+Q \sigma_{D}\left|\Phi_{0}\right|^{2} \Phi_{0}=0
$$

where $\sigma_{D}(\tau)=e^{-2 R \tau} \approx 1 /(1+2 R \tau)$. By substituting $\zeta(\xi, \tau)=\sigma_{D}(\tau) \xi, \chi(\tau)=\sigma_{D}(\tau) \tau$, and $\widehat{\Phi}=$ $\Phi_{0} \exp \left[i\left(R \sigma_{D}(\tau) \xi^{2} / 2 P\right)\right]$, we can transform Eq. (17) into the following standard NLSE:

$$
i \frac{\partial \widehat{\Phi}}{\partial \chi}+P \frac{\partial^{2} \widehat{\Phi}}{\partial \zeta^{2}}+Q|\widehat{\Phi}|^{2} \widehat{\Phi}=0
$$

The solution of the NLSE, given by Eq. (18), is localized both in position $\zeta$ and time $\chi$, which are equivalent respectively to the position and time variables $\xi$ and $\tau$. The resulting solution of the above equation then can be written as [32]:

$$
a(\xi, \tau)=\sqrt{\frac{2 P}{Q}}\left[\frac{4+16 i P \sigma_{D}(\tau) \tau}{1+16\left\{P \sigma_{D}(\tau) \tau\right\}^{2}+4\left\{\sigma_{D}(\tau) \xi\right\}^{2}}-1\right] \exp \left[-i\left\{\frac{R \sigma_{D}(\tau) \xi^{2}}{2 P}\right\}\right] \sqrt{\sigma_{D}(\tau)} .
$$




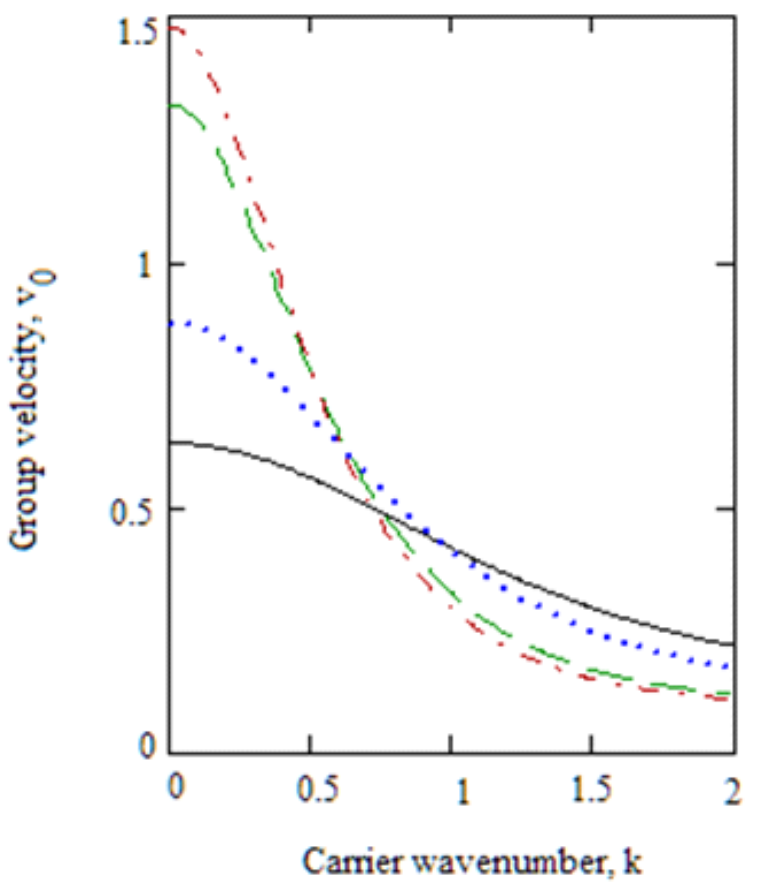

Figure 2. Variation of the group velocity $v_{0}$ with respect to the propagation wavenumber $k$ of the dust ion-acoustic wave for different values of the spectral index $\kappa$. The solid curve is for $\kappa=1.7$, the dotted curve is for $\kappa=2$, the dashed curve is for $\kappa=5$, and the dot-dashed curve is for $\kappa=50$. The other parameters of the plasma are the following: $\delta=n_{i 0} / n_{e 0}=2.2, \sigma=0.033, \beta_{d}=3.75 \times 10^{-6}$, and $\bar{\nu}_{i d}=0, \bar{\nu}_{i n}=0, \bar{\nu}_{d n}=0, \bar{\nu}_{L}=0$.

The above solution Eq. (19) predicts that the DIA wave is confined in a small region due to the nonlinear properties of the collisional dusty plasma. This solution is able to concentrate a significant amount of the wave energy into a relatively small area in space [32]. In the next section, the dissipative solution, Eq. (19), of the mNLSE, Eq. (12), is plotted against the position coordinate $\xi$ for a fixed value of $\tau$, taking $R$ (responsible for the dissipative effect) as a parameter.

Now, let us analyze the modulation instability of the dissipative DIA wave described by the mNLSE, Eq. (12), in the case of $P Q>0$, where $P$ and $Q$ are respectively the dispersive coefficient and nonlinear coefficient. We write the amplitude $a(\xi, \tau)$ as [31]:

$$
a(\xi, \tau)=\left[a_{0}+\delta a(\xi, \tau)\right] \exp \left[-i \int_{0}^{\tau} \Delta(\tau) d \tau-R \tau\right]
$$

where $\Delta$ is a possible nonlinear frequency shift, and $a_{0}$ (real constant) is the amplitude of the pump carrier wave. By expanding $\delta a(\xi, \tau)$ as

$$
\delta a(\xi, \tau)=(U+i W) \exp \left[i\left(K \xi-\int_{0}^{\tau} \Omega(\tau) d \tau\right)\right],
$$

with $U$ and $W$ respectively the real and imaginary parts of $\delta a(\xi, \tau)$. Now using Eqs. (12), (20) and (21) the following nonlinear dispersion relation for the modulation of the DIA wave is obtained:

$$
\Omega^{2}=\left(P K^{2}\right)^{2}\left(1-\frac{K_{c}^{2}(\tau)}{K^{2}}\right),
$$

where $K_{c}^{2}(\tau)=\left(2 Q\left|a_{0}\right|^{2} / P\right) \exp (-2 R \tau)$. From Eq. (22), the local-instability growth rate is obtained as

$$
\Gamma \equiv \operatorname{Im}[\Omega(\tau)]=\left|P K^{2}\right|\left(\frac{K_{c}^{2}(\tau)}{K^{2}}-1\right)^{1 / 2} .
$$




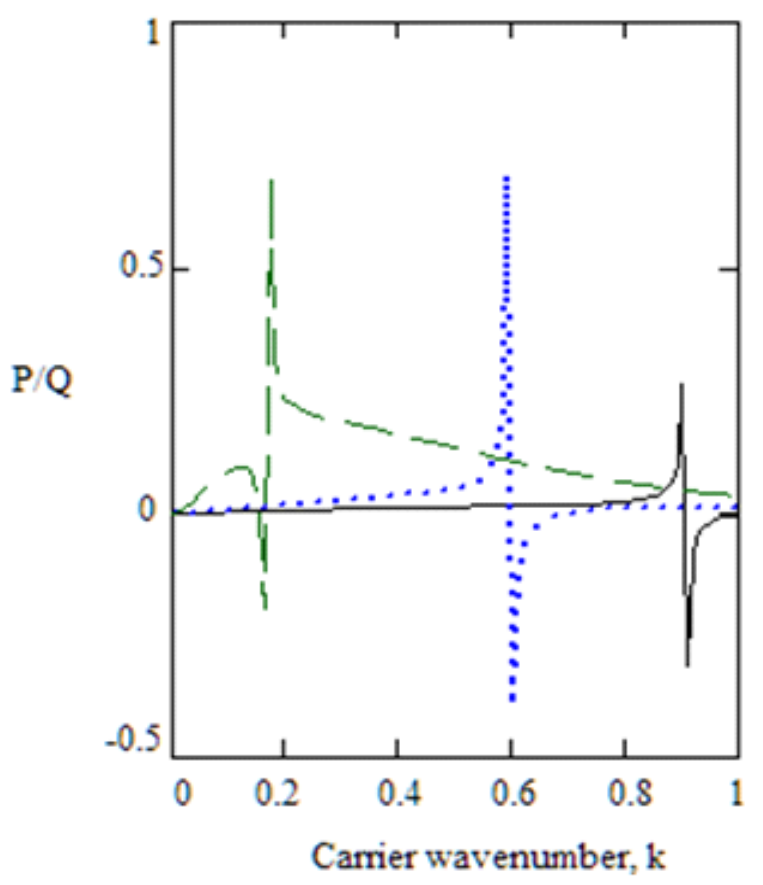

Figure 3. The variation of the ratio $P / Q$ of the coefficients of the dispersive and the nonlinear terms of the NLSE versus the carrier wavenumber $k$ for smaller values of the spectral index $\kappa$. The solid curve is for $\kappa=1.7$, the dotted curve is for $\kappa=1.8$, and the dashed curve is for $\kappa=2.2$. The other parameters of the plasma are the following: $\delta=n_{i 0} / n_{e 0}=2.2, \sigma=0.033, \beta_{d}=3.75 \times 10^{-6}, \bar{\nu}_{i d}=3.8 \times 10^{-2}, \bar{\nu}_{i n}=0.15, \bar{\nu}_{d n}=0.15, \bar{\nu}_{L}=5.4 \times 10^{-2}$, and $\Delta \sigma / \sigma_{0}=2$.

The instability growth will cease when

$$
\tau \geq \tau_{\max }=\frac{1}{2 R} \ln \left(\frac{2 Q\left|a_{0}\right|^{2}}{P K^{2}}\right) .
$$

Thus, we can say that $\tau_{\max }$ is the modulation instability period in the dissipative plasma. However, this instability period is absent in plasmas without dissipation.

\section{$5 \quad$ Results and Discussions}

In this section, we analyze the linear as well as nonlinear DIA wave dispersion relations. For the numerical analysis of our results, we consider the following typical parameters of the collisional dusty plasma $[27,33,34,35,36,37]: T_{e}=3 \mathrm{eV}, T_{i}=0.1 \mathrm{eV}, n_{i 0}=2 \times 10^{9} \mathrm{~cm}^{-3}, Z_{d}=75, m_{d} / m_{i}=2 \times 10^{7}$, $\nu_{L}=5.4 \times 10^{-2}, \nu_{i d}=3.8 \times 10^{-2}, \nu_{i n}=1.5 \times 10^{-1}, \nu_{d n}=1 \times 10^{-2}$, and $\kappa=1.6-10$.

Figure 1 shows the variation of the angular frequency $\omega$ with respect to the propagation wavenumber $k$ of the carrier DIA wave (the linear dispersion relation) for different spectral index $\kappa$ of the electron suprathermality (the solid curve is for $\kappa=1.7$, the dotted curve is for $\kappa=2$, the dashed curve is for $\kappa=5$ and the dot-dashed curve is for $\kappa=50$ ). It is seen that the angular frequency increases with the increase of the spectral index $\kappa$ of the electron suprathermality. However, this increase is significant only for the lower values of $\kappa$. We can see when $\kappa=5$ and $\kappa=50$, the change of angular frequency is not much, whereas when $\kappa=1.7$ and $\kappa=2$, there is a noticeable change in the angular frequencies. In this plot the effect of ionization, ion loss and collisions have been omitted.

Figure 2 shows the variation of the group velocity $v_{0}$ of the DIA wave with respect to the propagation wavenumber $k$ for different values of the spectral index $\kappa$ of the electron suprathermality. It is seen that 


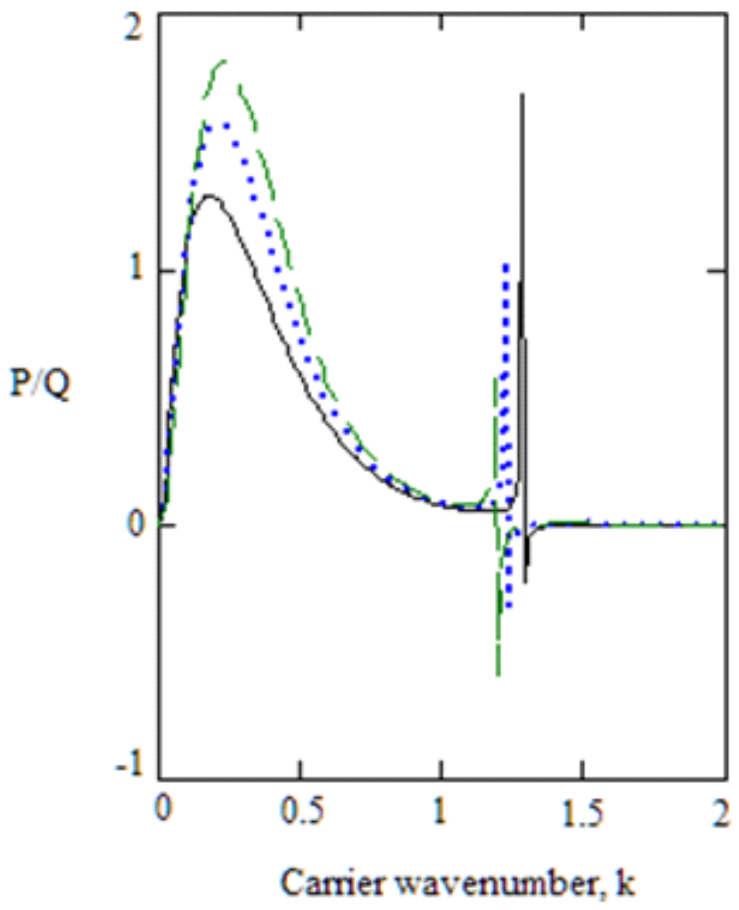

Figure 4. The variation of the ratio $P / Q$ of the coefficients of the dispersive and the nonlinear terms of the mNLSE versus the carrier wavenumber $k$ for comparatively larger values of the spectral index $\kappa$. The solid, dotted and the dashed curves are respectively for $\kappa=5, \kappa=10$, and $\kappa=50$. The other parameters of the plasma are the following: $\delta=n_{i 0} / n_{e 0}=2.2, \sigma=0.033, \beta_{d}=3.75 \times 10^{-6}, \bar{\nu}_{i d}=3.8 \times 10^{-2}, \bar{\nu}_{i n}=0.15, \bar{\nu}_{d n}=0.15$, $\bar{\nu}_{L}=5.4 \times 10^{-2}$, and $\Delta \sigma / \sigma_{0}=2$.

for comparatively longer wavelength perturbation, the group velocity increases by increasing the spectral index $\kappa$ while for comparatively shorter wavelengths (when $k>0.75$ (approximately)), the group velocity decreases with $\kappa$. It is noticeable that for larger values of $\kappa$ (like $\kappa=5$ and $\kappa=50$ ) the change in the group velocity is negligible. (Please see the dashed and the dot-dashed curves).

Figure 3 shows the variation of $P / Q$ (ratio of the dispersion coefficient and the nonlinear coefficient of the mNLSE, Eq. (12)) with respect to the propagation wavenumber $k$ for different values of spectral index $\kappa$. In this figure, the smaller values of $\kappa$ is considered (the solid curve is for $\kappa=1.7$, the dotted curve is for $\kappa=1.8$, and the dashed curve is for $\kappa=2.2$ ). It is observed that the critical wavenumber for the modulationally unstable regime to modulationally stable regime increases when spectral index $\kappa$ decreases. It is seen that as the value of the spectral index $\kappa$ increases, there is a shift of the peak of the curve (corresponding to the critical wavenumber) to the left in the wavenumber $k$ axis.

Figure 4 shows the variation of $P / Q$ with respect to the propagation wavenumber $k$ for comparatively larger values of the spectral index $\kappa$ (the solid curve is for $\kappa=5$, the dotted curve is for $\kappa=10$, and the dashed curve is for $\kappa=50$ ). It is observed that for comparatively larger values of the spectral index $\kappa$, there is no critical wavenumber in the range $0<k \leq 1$, but there is a critical wavenumber somewhere in the range $1<k<1.5$. It is also seen that there is no noticeable change of the critical wavenumber with the spectral index $\kappa$ for the modulationally unstable regime to modulationally stable regime, and the shift of the peak of the curve is also not noticeably changed.

Figure 5 shows the variation of the damping coefficient $R$ with respect to the propagation wavenumber $k$ for different values of the spectral index $\kappa$ (the solid curve is for $\kappa=1.6$, the dotted curve is for $\kappa=2$ and the dashed curve is for $\kappa=10$ ). It is seen that as the spectral index $\kappa$ increases then the damping coefficient $R$ decreases for longer wavelength perturbation. It is also found that when $\kappa=1.6$ and the propagation wavenumber $k$ in the range of $0<k<2$ then there is no change in the damping coefficient 


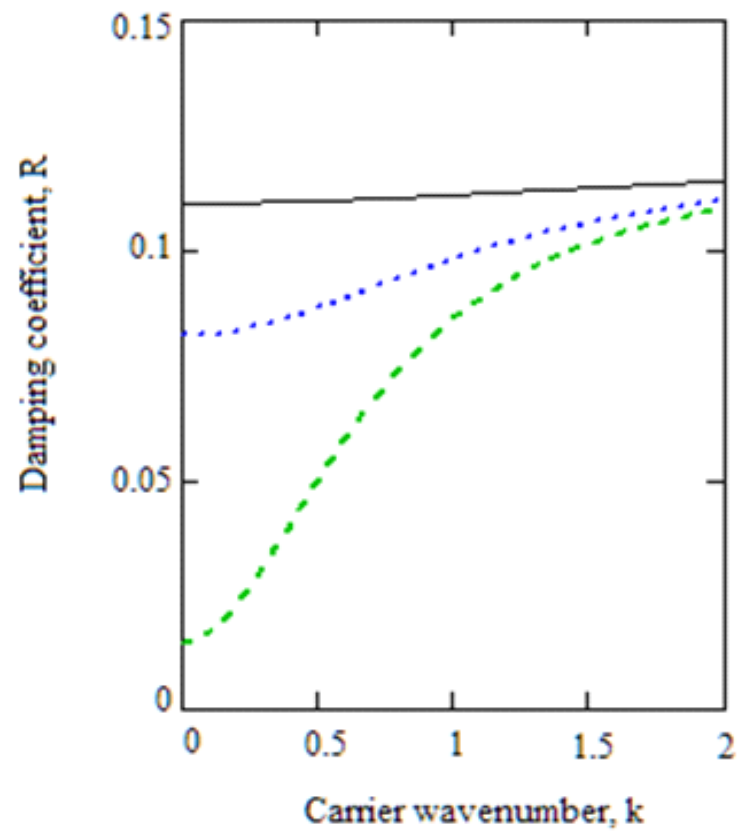

Figure 5. The variation of the damping coefficient $R$ of the mNLSE versus the carrier wavenumber $k$ for different $\kappa$. The solid curve is for $\kappa=1.6$, the dotted curve is for $\kappa=2$, and the dashed curve is for $\kappa=10$. The other parameters of the plasma are the following: $\delta=n_{i 0} / n_{e 0}=2.2, \sigma=0.033, \beta_{d}=3.75 \times 10^{-6}, \bar{\nu}_{i d}=3.8 \times 10^{-2}$, $\bar{\nu}_{i n}=0.15, \bar{\nu}_{d n}=0.15, \bar{\nu}_{L}=5.4 \times 10^{-2}$, and $\Delta \sigma / \sigma_{0}=2$.

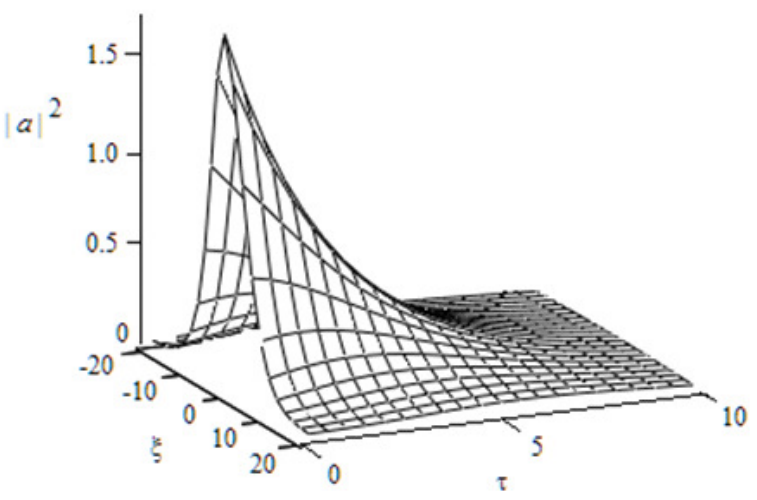

Figure 6. Variation of the modulus-square $|\Phi(\xi, \tau)|^{2}$ of the amplitude of the nonlinear dissipative DIA wave with respect to the position coordinate $\xi$, and time $\tau$, taking $\kappa=2$. The other parameters are the following: $k=0.7$, $\delta=n_{i 0} / n_{e 0}=2.2, \sigma=0.033, \beta_{d}=3.75 \times 10^{-6}, \bar{\nu}_{i d}=3.8 \times 10^{-2}, \bar{\nu}_{i n}=0.15, \bar{\nu}_{d n}=0.15, \bar{\nu}_{L}=0$, and $\Delta \sigma / \sigma_{0}=2$.

$R$ with respect to the wavenumber $k$, whereas for $\kappa=10$ the damping coefficient $R$ increases very fast with the increase of the wavenumber $k$. Thus, for larger values of the parameter $\kappa$ (called the spectral index), the damping coefficient is a sensitive function of the wavenumber $k$.

Figure 6 shows the modulus square of the amplitude of the nonlinear dissipative DIA wave, $|a|^{2}$, with respect to the position $\xi$ and time $\tau$ for the spectral index $\kappa=2$ where the ionization and ion loss are not considered. It is seen that the amplitude of the nonlinear DIA wave drastically falls with the increase of time $\tau$. This happens because there is no ionization whereas there are collisions among the different species. 


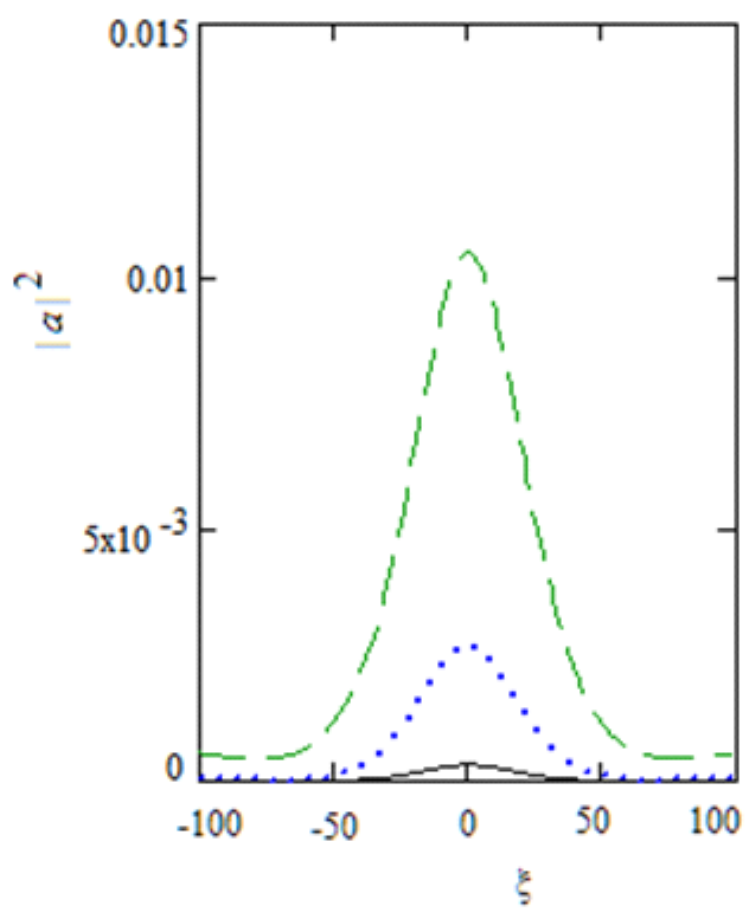

Figure 7. Variation of the modulus-square $|\Phi(\xi, \tau)|^{2}$ of the amplitude of the nonlinear dissipative DIA wave with respect to the position coordinate $\xi$, taking $\tau=40$ taking spectral index $\kappa$ as a parameter. The solid, dotted and the dashed curves are respectively for $\kappa=1.6, \kappa=1.7$, and $\kappa=2$. The other parameters are the following: $k=0.5$, $\delta=n_{i 0} / n_{e 0}=2.2, \sigma=0.033, \beta_{d}=3.75 \times 10^{-6}, \bar{\nu}_{i d}=3.8 \times 10^{-2}, \bar{\nu}_{i n}=0.15, \bar{\nu}_{d n}=0.15, \bar{\nu}_{L}=0$, and $\Delta \sigma / \sigma_{0}=2$.

Figure 7 shows the modulus square of the amplitude of the nonlinear dissipative DIA wave $|a|^{2}$ with respect to the position coordinate $\xi$ at a fixed time $\tau=40$ and for different values of the spectral index $\kappa$ (the solid curve is for $\kappa=1.6$, the dotted curve is for $\kappa=1.7$ and the dashed curve is for $\kappa=2$ ). It is seen that the amplitude of the nonlinear DIA wave drastically increases as the spectral index $\kappa$ increases.

Figure 8 shows the variation of the damping coefficient $R$ with respect to the propagation wavenumber $k$ for different values of the spectral index $\kappa$ (the solid curve is for $\kappa=1.6$, the dotted curve is for $\kappa=1.7$ and the dashed curve is for $\kappa=2$ ). In this figure, the collisions in the plasma system are omitted. Here collisions are omitted but ionization and ion loss are considered in the plasma. It is seen that the damping coefficient $R$ is very small, even for $\kappa=2$, at the very longer wavelength perturbation. It is important to mention here that the damping coefficient $R$ becomes negative indicating that instead of damping there can be instability for the cause of ionization.

\section{Conclusion}

In this work, we have studied the linear and nonlinear dispersions of a DIA wave propagating in a collisional dusty plasma along with the electron suprathermality and the dissipation effects due to the collisions among ion-dust, ion-neutral and dust-neutral particles. Here, a mNLSE is derived by using the standard reductive perturbation technique for the slow evolution of the DIA wave amplitude. We found that for typical parameters of the collisional dusty plasma, relevant to some astrophysical systems, the DIA wave amplitude is modulationally unstable below a certain value of the carrier wavenumber $k$. It is found from our numerical analysis that the carrier wavenumber and the suprathermality of electrons as well as the ionization and ion loss have profound effects on the dispersion properties of the DIA wave. Finally, we would like to emphasize that the results obtained from the current investigation presented in this paper may be useful to explain modulation instability and envelope soliton excitations of DIA waves in some astrophysical and space plasmas where superthermal electrons are present. 


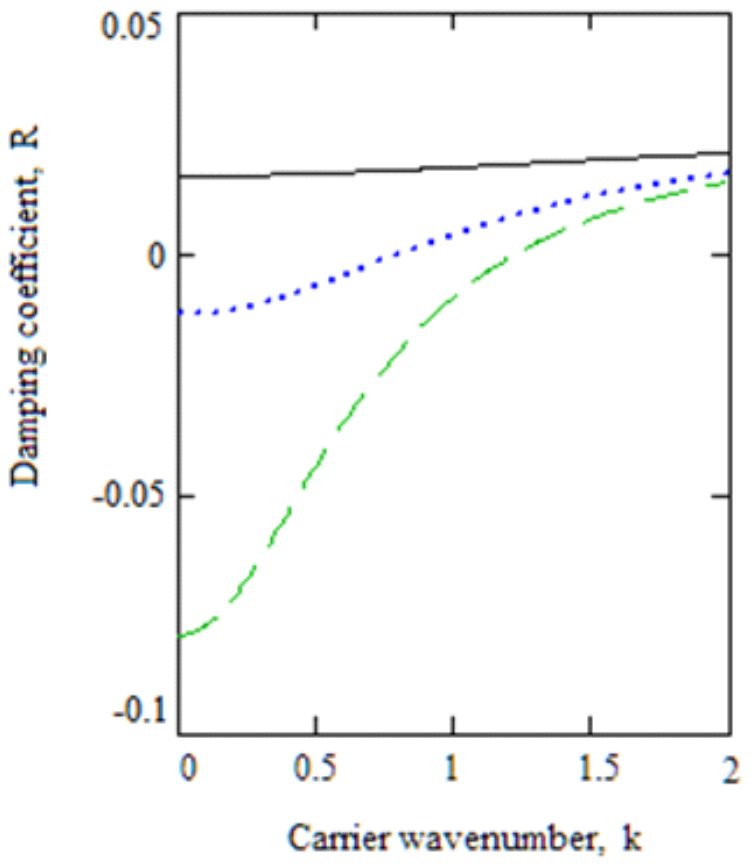

Figure 8. The damping coefficient $R$ with respect to the carrier wave number $k$ taking the spectral index $\kappa$ as a parameter. The solid, dotted and the dashed curves are respectively for $\kappa=1.6, \kappa=1.7$, and $\kappa=2$. The other parameters of the plasma are the following: $k=0.5, \delta=n_{i 0} / n_{e 0}=2.2, \sigma=0.033, \beta_{d}=3.75 \times 10^{-6}, \bar{\nu}_{i d}=0.0$, $\bar{\nu}_{i n}=0.0, \bar{\nu}_{d n}=0.0, \bar{\nu}_{L}=5.4 \times 10^{-2}$, and $\Delta \sigma / \sigma_{0}=2$.

\section{Appendix A}

The parameters $f_{1}, f_{2}, f_{3}, f_{4}, g_{1}, g_{2}, g_{3}, g_{4}$ and $h$ appearing Eqs. (13)-(15) during the derivation of the mNLSE are listed below:

$$
\begin{gathered}
f_{1}=k\left[a_{1} a_{21}+a_{2} a_{33}+a_{2} a_{20}+a_{1} a_{34}\right], \\
g_{1}=v_{0} a_{9}-a_{10}, \\
f_{2}=k\left[a_{2} a_{21}+a_{2} a_{34}\right], \\
g_{2}=v_{0} a_{10}, \\
f_{3}=k\left[a_{3} a_{23}+a_{3} a_{36}+a_{4} a_{22}+a_{4} a_{35}\right], \\
g_{3}=v_{0} a_{11}-a_{12}, \\
f_{4}=-\omega\left(-a_{3} a_{23}+a_{3} a_{36}+2 a_{4} a_{22}\right)+\omega a_{3}^{2} a_{4} \\
+2 k\left(a_{4} a_{23}+a_{4} a_{36}\right)+k a_{4}^{2} a_{3}-k \sigma_{i}\left(a_{3} a_{22}+a_{3} a_{35}\right), \\
g_{4}=v_{0} a_{12}-\sigma_{i} a_{11}, \\
h=a_{19}+a_{32},
\end{gathered}
$$

where $a_{1}, a_{2}, a_{3}, a_{4}, b_{1}, b_{2}, b_{3}, b_{4}$ are defined earlier and the remaining other parameters are defined below:

$$
\begin{gathered}
a_{5}=v_{0} a_{1}-a_{2}, \\
a_{6}=v_{0} a_{2}+\beta_{d}, \\
a_{7}=v_{0} a_{3} a_{4},
\end{gathered}
$$




$$
\begin{aligned}
& a_{8}=v_{0} a_{4}-\sigma_{i} a_{3}-1, \\
& a_{9}=\frac{\omega a_{5}+k a_{6}}{\omega^{2}}, \\
& a_{10}=\frac{v_{0} a_{2}+\beta_{d}}{\omega}, \\
& a_{11}=\frac{\omega a_{7}+k a_{8}}{\omega^{2}-k^{2} \sigma_{i}}, \\
& a_{12}=\frac{\omega}{k} a_{11}-\frac{a_{7}}{k}, \\
& a_{13}=-k a_{1} a_{2}, \\
& a_{14}=\frac{k^{2}}{2} a_{2}^{2}, \\
& a_{15}=-k a_{3} a_{4}, \\
& a_{16}=\frac{1}{2}\left[\omega a_{3} a_{4}-2 k a_{4}^{2}+k \sigma_{i} a_{3}^{2}\right] \text {, } \\
& a_{17}=-\frac{1}{\omega^{2}}\left[\omega a_{13}-k a_{14}\right], \\
& a_{18}=\frac{-1}{\omega^{2}-k^{2} \sigma_{i}}\left[\omega a_{15}+k a_{16}\right] \text {, } \\
& a_{19}=\frac{\beta+a_{17}(\delta-1)-a_{18} \delta}{a_{3} \delta-a_{1}(\delta-1)-\left(4 k^{2}+\alpha\right)}, \\
& a_{20}=a_{1} a_{19}+a_{17}, \\
& a_{21}=\frac{\omega}{k} a_{20}+\frac{a_{13}}{k}, \\
& a_{22}=a_{3} a_{19}+a_{18} \text {, } \\
& a_{23}=\frac{\omega}{k} a_{22}+\frac{a_{15}}{k}, \\
& a_{24}=-2 a_{1} a_{2} \text {, } \\
& a_{25}=a_{2} \text {, } \\
& a_{26}=-2 a_{3} a_{4}, \\
& a_{27}=-\omega a_{4} a_{11}+\omega a_{3} a_{12}+v_{0} a_{3} a_{4}-2 a_{4}^{2}+a_{3}^{2} \sigma_{i}, \\
& a_{28}=\frac{1}{v_{0}^{2}}\left[a_{25}-v_{0} a_{24}\right], \\
& a_{29}=\frac{1}{v_{0}} a_{25}, \\
& a_{30}=\frac{a_{27}+v_{0} a_{26}}{\sigma_{i}-v_{0}^{2}}, \\
& a_{31}=v_{0} a_{30}+a_{26}, \\
& a_{32}=\frac{2 \beta-a_{30} \delta+a_{28}(\delta-1)}{-\alpha-\frac{\delta}{\sigma_{i}-v_{0}}+\frac{\beta_{d}(\delta-1)}{v_{0}^{2}}}, \\
& a_{33}=\frac{\beta_{d}}{v_{0}^{2}} a_{32}+a_{28}, \\
& a_{34}=\frac{\beta_{d}}{v_{0}} a_{32}+a_{29}, \\
& a_{35}=\frac{-a_{32}}{\sigma_{i}-v_{0}}+a_{30}, \\
& a_{36}=\frac{-v_{0}}{\sigma_{i}-v_{0}} a_{32}+a_{31} \text {. }
\end{aligned}
$$




\section{References}

1. D. A. Mendis and M. Rosenberg,, "Cosmic dusty plasma" Annu. Rev. Astron. Astrophys. 32, 419 (1994).

2. M. Horányi, D. A. Mendis, "The dynamics of charged dust in the tail of comet Giacobini-Zinner", J. Geophys. Res. 91, 355 (1986).

3. M. Horányi, "Charged dust dynamics in the solar system" Annu. Rev. Astrophys. 34, 383 (1996).

4. F. Verheest, Waves in Dusty Space Plasmas (Kluwer Academic Publishers, Dordrecht, 2000).

5. P. K. Shukla, "A survey of dusty plasma physics" Phys. Plasmas 8, 1791 (2001).

6. P. K. Shukla, A. A. Mamun, Introduction to Dusty Plasma Physics (Institute of Physics Publishing Ltd., Bristol, 2002).

7. A. Barkan, R. L. Merlino, N. D'Angelo, "Laboratory observation of the dust-acoustic wave mode" Phys. Plasmas 2, 3563 (1995).

8. A. Barkan, N. D'Angelo, R. L. Merlino, "Experiments on ion-acoustic waves in dusty plasmas" Planet. Space Sci. 44, 239 (1996).

9. R. L. Merlino, A. Barkan, C. Thompson, N. D'Angelo, "Laboratory studies of waves and instabilities in dusty plasmas" Phys. Plasmas 5, 1607 (1998).

10. A.Homann, A. Melzer, S. Peters, A. Piel, "Determination of the dust screening length by laser-excited lattice waves" Phys. Rev. E 56, 7138 (1997).

11. P. K. Shukla, B. Eliasson, "Colloquium: Fundamentals of dust-plasma interactions" Rev. Mod. Phys. 81, 25 (2009).

12. V. N.Tsytovich, G. E. Morfill, S. V. Vladimirov, H. M.Thomas, Elementary Physics of Complex Plasmas (Springer, Berlin, 2008).

13. V. E. Fortov, A. V. Ivlev, S. A. Khrapak, A. G. Khrapak, G. E. Morfill, "Complex (dusty) plasmas: Current status, open issues, perspectives" Phys. Reports 421, 1 (2005).

14. P. V. Bliokh, V. V. Yaroshenko, "Electrostatic waves in Saturn's rings" Sov. Astron. (Engl. Transl.) 29, 330 (1985).

15. U. de Angelis, V. Formisano, M. Giordano, "Ion plasma waves in dusty plasmas: Halley's comet" J. Plasma Phys. 40, 399 (1988).

16. P. K. Shukla, L. Stenflo, "Stimulated scattering of electromagnetic waves in dusty plasmas" Astrophys. Space Sci. 190, 23 (1992).

17. N. N. Rao, Shukla P. K., Yu M. Y., "Dust-Acoustic Waves in Dusty Plasmas" Planet. Space Sci. 38, 543 (1990).

18. P. K. Shukla, V. P. Silin,"Dust Ion Acoustic Wave" Physica Scripta 45, 508 (1992).

19. F. Melands $\varnothing$," Lattice waves in dust plasma crystals" Phys. Plasmas 3, 3890 (1996).

20. Y. Nakamura, H. Bailung, P. K. Shukla,"Observation of ion-acoustic shocks in a dusty plasma" Phys. Rev. Lett. 83, 1602 (1999).

21. R. L. Merlino , J. Goree,"Dusty Plasmas in the Laboratory, Industry, and Space", Phys. Lett. A 332, 82 (2004).

22. M. Salimullah, "Low-frequency dust-lower-hybrid modes in a dusty plasma", Phys. Lett. A 215, 296 (1996).

23. R. Bharuthram, P. K. Shukla, "Large amplitude ion-acoustic solitons in a dusty plasma", Planet. Space Sci. 40, 973 (1992).

24. S. I. Popel, M. Y. Yu, "Ion Acoustic Solitons in Impurity-Containing Plasmas", Contrib. Plasma Phys. 35, $103(1995)$

25. Y. Nakamura, H. Bailung, P. K. Shukla, "Observation of Ion-Acoustic Shocks in a Dusty Plasma", Phys. Rev. Lett. 83, 1602 (1999).

26. A. A. Mamun, P. K. Shukla, "The role of dust charge fluctuations on nonlinear dust ion-acoustic waves", IEEE Trans. Plasma Sci. 30, 720 (2002).

27. S. Mayout, L. A. Gougam, M. Tribeche, "Effects of ionization and ion loss on dust ion-acoustic solitary waves in a collisional dusty plasma with suprathermal electrons", Phys. Plasmas 23, 033701 (2016).

28. T. Tanuiti, "Reductive perturbation methods and farfields of wave equations", Prog. Theor. Phys. Suppl. 55, 1-35 (1974).

29. X. Ju-Kui, "Modulational Instability of Dust Ion Acoustic Waves in a Collisional Dusty Plasma", Commun. Theor. Phys. 40, 717 (2003).

30. A. Hasegawa, "Plasma instabilities and nonlinear effects" Nonlinear Effects and Plasma Instabilities Chap. 4. (Springer-Verlag, Berlin, 1975)

31. G. Shimin, M. Liquan, "Modulation instability and dissipative rogue waves in ion-beam plasma: Roles of ionization, recombination, and electron attachment", Phys. Plasmas 21, 112303 (2014).

32. W. M. Moslem, R. Sabry, S. K. El-Labany, P. K. Shukla,"Dust-acoustic rogue waves in a nonextensive plasma", Phys. Rev. E84, 066402 (2011). 
33. N. D'Angelo, "Ionization instability in dusty plasmas", Phys. Plasmas 4, 3422 (1997).

34. D. Samsonov, G. Goree, "Instabilities in a dusty plasma with ion drag and ionization", Phys. Rev. E 59, 1047 (1999).

35. X. Wang, A. Bhattacharjee, S. K. Guo, J. Goree, "Ionization instabilities and resonant acoustic modes", Phys. Plasmas 8, 5018 (2001).

36. G. Praburam, J. Goree, "Experimental observation of very low-frequency macroscopic modes in a dusty plasma", Phys. Plasmas 3, 1212 (1996).

37. P. K. Shukla, G. E. Morfill, "Ionization instability of dust-acoustic waves in weakly ionized colloidal plasmas", Phys. Lett. A 216, 153 (1996). 